\title{
Trabalho rural e intoxicações por agrotóxicos
}

\section{Rural work and pesticide poisoning}

\footnotetext{
1 Faculdade de Medicina, Universidade Federal de Pelotas, Pelotas, Brasil.

Correspondência N. M. X. Faria Rua República 80/1401, Bento Gonçalves, RS 95700-000, Brasil. neicef@italnet.com.br
}

\begin{abstract}
Pesticide use is intensive in Brazilian agriculture. Population-based studies on the characteristics of pesticide use and pesticide poisoning are scarce. This study describes the profile of occupational exposure and pesticide poisoning incidence. Farm characteristics and pesticide occupational exposure were evaluated using a cross-sectional design. Among 1,379 farmers/ farm workers, annual incidence of pesticide poisoning was 2.2 episodes per 100 exposed. Based on Poisson regression, applying pesticide, reentering crop fields after spraying, and working with pesticides on more than one farm were the types of exposure that presented a positive correlation with pesticide poisoning. The results may be useful for planning activities aimed at reducing occupational pesticide poisoning among rural workers.
\end{abstract}

Pesticides; Pesticide Exposure; Occupational Health; Incidence
Neice Müller Xavier Faria 1

Luiz Augusto Facchini 1

Anaclaudia Gastal Fassa 1

Elaine Tomasi 1

O uso de agrotóxicos na agricultura é intensivo, multiquímico e várias publicações têm apontado as intoxicações por agrotóxicos como um grave problema de saúde, especialmente entre trabalhadores rurais 1,2,3. Contudo, são escassos os estudos brasileiros de base populacional sobre as características do uso ocupacional ou sobre as intoxicações por agrotóxicos 4,5.

Nos Estados Unidos, um estudo avaliou os bancos de dados oficiais sobre mortalidade, internações hospitalares e centros de intoxicações durante um período de seis anos. Os pesticidas foram responsáveis por 341 mortes, 25.418 hospitalizações e 338.170 casos de intoxicações 6 , representando 40,0\% do total das intoxicações 7 . Em países do terceiro mundo vários estudos apontam as intoxicações por pesticidas como um dos principais problemas de saúde, representando $15 \%$ do conjunto das intoxicações na Costa Rica 8, 9,0 a 13,0\% das intoxicações na África do Sul 9 e 6,0\% das mortes registradas em hospitais públicos no Sri Lanka ${ }^{3}$. Apesar da importância das intoxicações por agrotóxicos, os índices de sub-registro são enormes. Na Nicarágua, onde é obrigatória a notificação, apenas $35,0 \%$ dos casos atendidos em serviços de saúde e $17,0 \%$ do total populacional dos casos foram registrados 2 .

No Brasil, entre 1997 a 2000 houve um aumento médio de $18,0 \%$ nas vendas de agrotóxi- 
cos, com destaque para os herbicidas, cujas vendas cresceram 31,0\% (http://www.sindag. com.br, acessado em 21/Mar/2002). Em 2000, segundo dados do Sistema Nacional de Informações Tóxico-Farmacológicas (SINITOX - Casos Registrados de Intoxicação Humana e Envenenamento. Análise do Ano de 2000. Fundação Oswaldo Cruz), os pesticidas de uso agrícola foram responsáveis por 7,0\% das intoxicações e 37,0\% dos óbitos por intoxicações. Ao se agruparem estes casos com aqueles causados por pesticidas de uso doméstico, produtos veterinários e raticidas, os pesticidas se tornam responsáveis por $17,0 \%$ dos casos e $57,0 \%$ dos óbitos por intoxicações. Dentre estas intoxicações, 13,0\% ocorreram após exposições ocupacionais; porém, num tema conhecido pelo alto sub-registro, tais dados são apenas a parte visível do problema dos agrotóxicos, referindo-se quase sempre aos episódios mais graves 10 .

Em artigos anteriores foram apresentados: uma caracterização geral das condições de trabalho e da saúde dos trabalhadores rurais $4 \mathrm{e}$ uma abordagem analítica dos fatores relacionados com a saúde mental dos agricultores 11 . O presente artigo tem como objetivos descrever as características da exposição ocupacional aos agrotóxicos e analisar a incidência de intoxicações por agrotóxicos, no contexto da agricultura familiar.

\section{Metodologia}

Este estudo, de delineamento transversal, foi desenvolvido entre os trabalhadores rurais dos municípios de Antônio Prado e Ipê, na Serra Gaúcha. Esta região é caracterizada por propriedades familiares médias e pequenas (37ha em média), diversidade de modelos de produção agrícola (incluindo agricultores ecológicos) e predomínio da fruticultura, particularmente uva e maçã. Considerou-se trabalhador rural quem trabalhava no mínimo 15 horas por semana em atividades agrícolas (Fundação Instituto Brasileiro de Geografia e Estatística. Pesquisa Nacional por Amostras de Domicílios; 1992), sendo entrevistadas as pessoas que tinham 15 anos ou mais. A definição de agrotóxicos usada neste estudo está descrita na Lei Federal no 7.802/89 (Ministério do Trabalho e Previdência Social. Intoxicaçôes Agudas e Crônicas Produzidas pelos Agrotóxicos: Normas Técnicas para Avaliação da Incapacidade; 1991), estando incluídos nesta definição os produtos usados como inseticidas, fungici- das, herbicidas e pesticidas de uso veterinário.

A amostra utilizada conferiu um poder estatístico de $80 \%$ e um nível de confiança de $95 \%$ para a análise das associações, com um risco relativo mínimo de 1.70, entre as características sócio-demográficas e ocupacionais e as intoxicações por agrotóxicos. O questionário foi prétestado e aperfeiçoado em estudo piloto. O controle de qualidade incluiu a re-visita de $10,0 \%$ das propriedades, sendo realizada uma segunda entrevista de pelo menos um trabalhador rural. A concordância em relação aos principais aspectos do questionário foi avaliada pelo teste kappa.

Todos os dados foram obtidos com base na informação referida dos trabalhadores, entrevistados durante o verão de 1996 (safra). Foi utilizado um questionário para cada estabelecimento e outro individual, que captavam características sócio-demográficas, estrutura agrária, produção agrícola, jornadas de trabalho, formas de exposição química, uso de Equipamentos de Proteção Individual (EPI) e as intoxicações por agrotóxicos. Os dados sobre uso de EPI foram obtidos apenas para os trabalhadores expostos aos agrotóxicos. Para os indicadores econômicos (renda bruta da produção e nível de mecanização) foram construídas escalas descritas em artigo anterior 11.

O uso intensivo na agricultura foi obtido excluindo os estabelecimentos que usavam somente formicidas (uso ocasional), ou produtos veterinários (em geral injetáveis ou usados em banhos para gado) ou ainda o sulfato de cobre (usado também por agroecologistas).

A definição de intoxicação por agrotóxicos foi obtida de duas maneiras: ocorrência de alguma intoxicação ocupacional nos 12 meses anteriores à entrevista e a informação do trabalhador sobre a existência de algum episódio de intoxicação ao longo da vida.

Os casos de intoxicação nos últimos 12 meses foram caracterizados quanto à gravidade, tipo químico envolvido, circunstâncias do acidente, tempo de afastamento, assistência recebida e seqüelas e emissão da Comunicação de Acidentes de Trabalho (CAT). As diferenças entre grupos foram avaliadas pelos testes estatísticos qui-quadrado e tendência linear.

A partir dos episódios de intoxicações ao longo da vida e dos anos de exposição aos agroquímicos, construiu-se uma estimativa da taxa bruta de incidência de intoxicações, para cada 100 trabalhadores por ano de exposição química 2,12. Com o programa Stata 8.0, foi desenvolvida a análise multivariada que usou regressão de Pois- 
son levando em consideração o tempo de exposição química. Devido à superdispersão do desfecho, foi utilizada a regressão de Poisson com variância robusta. Os fatores de confusão selecionados pelo critério de p menor ou igual a 0,20.

Os indicadores agroeconômicos foram construídos valendo-se de dados do estabelecimento. Como a presença de empregados era maior nos estabelecimentos mais ricos, estes ficavam com indicadores de renda superiores à média dos proprietários e arrendatários. Por esta razão, os empregados foram excluídos da análise multivariada, que ficou restrita aos $93,0 \%$ que eram proprietários ou arrendatários.

\section{Resultados}

No verão de 1996 foram entrevistados 1.479 trabalhadores rurais em 495 unidades produtivas (estabelecimentos). As perdas e as recusas representaram 5,0\% dos elegíveis.

Avaliando segundo características demográficas e relações de trabalho, o sexo masculino correspondia a 55,0\% dos proprietários e dos arrendatários e a 68,0\% dos empregados $(\mathrm{p}=$ 0,02). A idade média dos proprietários e arrendatários foi cerca de 42 anos e entre empregados, cerca de 33 anos. O nível de escolaridade (média de 4,8 anos) foi similar nos três grupos.

Características da exposição ocupacional aos agrotóxicos $(\mathrm{n}=1.479)$

Dentre os estabelecimentos, 95,0\% informaram usar algum tipo de agrotóxico e 73,0\% faziam uso regular e intensivo de agrotóxicos na agricultura (355 estabelecimentos). Os tipos químicos mais utilizados em ambas situações encontram-se na Tabela 1.

Em média, 75,0\% dos trabalhadores rurais relataram trabalhar regularmente com agrotóxicos $(n=1.105)$. Nas 355 propriedades com uso intensivo de agrotóxicos, esta proporção era de $86,0 \%$. Nestas propriedades de uso intensivo o tempo médio individual de exposição química foi de 16,0 anos $(\mathrm{dp}=11,7)$.

A prevalência de exposição agroquímica foi maior entre os homens $(86,0 \%)$ do que entre as mulheres $(68,0 \%)(\mathrm{p}<0,001)$. No grupo de $30 \mathrm{a}$ 49 anos, $87,0 \%$ dos trabalhadores lidavam com agrotóxicos. Nas demais faixas etárias a proporção de expostos era igual ou inferior à média ( $\mathrm{p}<0,001)$. Os trabalhadores com escolaridade média relataram maior exposição $(83,0 \%$ no grupo com 5 a 8 anos de escola); os analfabetos relataram a menor proporção de trabalho com agrotóxicos: $58,2 \%$ ( $p<0,001)$. Os tra- balhadores com maior exposição aos pesticidas recebiam mais orientações técnicas para práticas agrícolas.

Avaliando segundo indicadores econômicos, observou-se maior intensidade de aplicação de agrotóxicos entre os que trabalhavam em propriedades com maior renda bruta da produção, maior nível de mecanização e área de 25 a 100ha $(\mathrm{p}<0,05)$.

Os homens apresentaram maior proporção de exposição química em todas as formas de exposição exceto lavar roupa com agrotóxicos ( $\mathrm{p}<0,001$ ). Assim, respectivamente, 83,0\% dos homens e $51,0 \%$ das mulheres aplicavam pesticidas, $80,0 \%$ e $39,0 \%$ preparavam a calda, $56,0 \%$ e $37,0 \%$ re-entravam em locais com aplicação recente, $8,0 \%$ e $2,0 \%$ trabalhavam com agrotóxicos em mais de um estabelecimento e $17,0 \%$ e $71,0 \%$ lavavam roupas contaminadas.

Comparados aos proprietários, os empregados tinham menor exposição química em tratamentos veterinários, mas trabalhavam com mais freqüência em outras propriedades $(\mathrm{p}<$ $0,001)$. Não foram evidenciadas diferenças nas demais formas de exposição.

Quanto maior a jornada de trabalho agrícola, na safra ou fora da safra, maior a exposição aos agrotóxicos $(\mathrm{p}<0,01)$.

Equipamentos de Proteção Individual ( $\mathrm{n}=1.105$ expostos aos agrotóxicos)

Considerando os equipamentos mais específicos para proteção química, mais de $35,0 \%$ dos trabalhadores admitiram nunca usar luvas, máscaras ou roupas de proteção.

O uso de EPI foi mais freqüente entre os homens e entre as pessoas com escolaridade média - 5 a 8 anos (Tabela 2). O grupo sem escolaridade era o que menos usava estes equipamentos. Verificou-se que o uso destas medidas de proteção era reduzido entre os agricultores mais idosos $(p<0,03)$. O acesso a orientações técnicas para práticas agrícolas mostrou-se relacionado a maior uso de EPI específico para proteção química.

O uso de EPI era menor entre os empregados $(\mathrm{p}<0,02)$. Os trabalhadores rurais que usavam mais EPI trabalhavam nos estabelecimentos com maior renda bruta de produção $(\mathrm{p}<0,02)$, maior nível de mecanização ( $\mathrm{p}<0,001)$ e tinham jornada de trabalho agrícola mais extensa $(\mathrm{p}<0,001)$.

Para a maioria das formas de exposição, o uso de todos os EPI crescia linearmente conforme aumento da exposição (Tabela 2). A única exceção foi lavar roupas contaminadas com agrotóxicos, em que a associação foi inversa.

Intoxicações ocupacionais por agrotóxicos 
Dentre os 1.479 entrevistados, foram identificados, nos 12 meses anteriores à entrevista, 145 trabalhadores com algum tipo de acidente de trabalho. As intoxicações por agrotóxicos corresponderam a $16,0 \%$ destes acidentes (23 casos). Ou seja, 2,0\% dos 1.105 agricultores que trabalhavam com agrotóxicos tiveram intoxicações por estes produtos.

As intoxicações ocorreram entre outubro e janeiro em $80,0 \%$ das vezes. De acordo com o entrevistado, a gravidade destas intoxicações foi considerada como leve-moderada em $80,0 \%$ dos casos e como grave em $20,0 \%$ dos casos (nenhum com risco imediato para a vida).

Com relação ao impacto na produtividade, $32,0 \%$ dos intoxicados interromperam o trabalho de um a sete dias, $12,0 \%$ de 8 a 15 dias e $4,0 \%$ por mais de 15 dias. Os restantes $52,0 \%$ não pararam o trabalho, apenas trocaram para outras atividades mais leves.

Os produtos responsáveis pelo maior número de ocorrências foram fungicidas $(28,0 \%)$, herbicidas $(16,0 \%)$, inseticidas/formicidas $(8,0 \%)$ e produtos veterinários (8,0\%). Em $20,0 \%$ dos casos não foi identificado o produto causador da intoxicação e em 16,0\% dos casos houve envolvimento de dois ou mais produtos simultaneamente. Entre os fungicidas destacaram-se os ditiocarbamatos (que de forma isolada ou associada foram referidos em $28,0 \%$ dos casos) e entre os herbicidas os principais produtos identificados foram o glifosate $(16,0 \%$ das intoxicações) e o paraquat (8,0\% dos casos).

Em 48,0\% dos casos o tratamento foi exclusivamente caseiro e em $20,0 \%$ foi necessário buscar assistência hospitalar. Em quatro casos $(17,0 \%)$ o intoxicado permanecia com algum tipo de seqüela em conseqüência do acidente ocorrido.

Em apenas um acidente $(4,0 \%)$ foi emitida a CAT, isto é, neste tipo de acidente o sub-registro na fonte oficial foi da ordem de $96,0 \%$. Nenhum destes casos de intoxicações foi notificado ao SINITOX (Centro de Informações Toxicológicas - Rio Grande do Sul). Não houve registro de óbito devido à intoxicação por agrotóxicos no período avaliado.

Intoxicações por agrotóxicos ao longo

da vida - fatores associados $(\mathrm{n}=1.379)$

Dentre os entrevistados, $12,0 \%$ relataram pelo menos um episódio de intoxicação ao longo de sua vida. O diagnóstico foi estabelecido por médicos em $58,0 \%$ dos casos, pelo próprio entrevistado em $36,0 \%$ dos casos e por outras pessoas em $6,0 \%$ dos casos. Comparando com a en-
Tabela 1

Tipos químicos mais usados no total dos estabelecimentos e naqueles com uso intensivo de agrotóxicos na agricultura. Antônio Prado e Ipê, Rio Grande do Sul, Brasil, 1996.

\begin{tabular}{lcc}
\hline Tipos químicos & \multicolumn{2}{c}{ Estabelecimentos rurais $-\%$ de uso } \\
& Total $(n=495)$ & Uso intensivo ( $=355)$ \\
\hline Fungicidas - geral & 69,3 & 86,8 \\
Sulfato de cobre & 61,1 & 75,5 \\
Ditiocarbamato & 52,7 & 72,4 \\
Alaninatos & 25,3 & 34,9 \\
Benzimidazois & 23,7 & 32,7 \\
Ftalimidas & 17,3 & 23,9 \\
Dodine - Guanidinas & 12,7 & 17,5 \\
Inseticidas - geral & 70,8 & 86,9 \\
Fosforados - total & 81,6 & 91,2 \\
Fosforados na agricultura & 42,6 & 58,6 \\
Fenthion (OF) & 17,4 & 23,9 \\
Dimetoato (OF) & 8,2 & 11,3 \\
Piretróides na agricultura & 10,2 & 14,1 \\
Herbicidas - geral & 53,9 & 74,1 \\
Glifosate & 46,5 & 63,9 \\
Triazinas* & 40,7 & 47,7 \\
Paraquat & 14,1 & 19,4 \\
& &
\end{tabular}

* usadas também como inseticidas.

trevista original, o controle de qualidade mostrou boa concordância (kappa $=0,66$ para intoxicações) e uma maior proporção de intoxicações.

Em relação aos proprietários, a prevalência de intoxicações dos empregados fixos era semelhante, entretanto, o grupo dos empregados temporários mostrou prevalência quase três vezes maior. A análise dos fatores associados às intoxicações, apresentada a seguir, exclui os empregados e refere-se a uma amostra de 1.379 proprietários e arrendatários.

A incidência anual de intoxicações por agrotóxicos foi de 2,2 episódios por cem trabalhadores expostos, não havendo diferenças conforme sexo (Tabela 3 ).

Os trabalhadores com idade acima de quarenta anos apresentaram taxas de incidência menores que o grupo mais jovem. Contudo, a associação entre idade e intoxicação não foi significativa. Não foram evidenciadas diferenças significativas segundo indicadores sócioeconômicos como escolaridade, renda bruta da produção, nível de mecanização, área, posse de automóvel ( $p=0,09)$, tipos de culturas e de rebanhos animais (Tabela 3).

Avaliando segundo exposição ocupacional aos agrotóxicos, o grupo que parou de usar agrotóxicos há mais de um ano apresentou o 
Uso de equipamentos de proteção, entre trabalhadores expostos aos pesticidas, conforme dados sócio-demográficos e acesso a informações. Antônio Prado e Ipê, Rio Grande do Sul, Brasil, 1996.

\begin{tabular}{|c|c|c|c|c|}
\hline Variáveis & $\mathbf{n}$ & $\begin{array}{c}\text { Luvas } \\
(\%)\end{array}$ & $\begin{array}{c}\text { Máscaras } \\
(\%)\end{array}$ & $\begin{array}{c}\text { Roupas de proteção } \\
(\%)\end{array}$ \\
\hline Sexo & & $\mathrm{p}<0,001$ & $\mathrm{p}<0,001$ & $\mathrm{p}<0,001$ \\
\hline Masculino & 706 & 62,8 & 60,0 & 67,8 \\
\hline Feminino & 399 & 45,2 & 37,2 & 55,6 \\
\hline Idade em anos & & $\mathrm{p}=0,006^{\star}$ & $\mathrm{p}<0,001$ & $\mathrm{p}=0,004$ \\
\hline $15-19$ & 88 & 63,2 & 46,0 & 66,7 \\
\hline $20-29$ & 179 & 56,8 & 54,0 & 63,1 \\
\hline $30-39$ & 284 & 61,3 & 62,8 & 70,6 \\
\hline $40-49$ & 256 & 56,2 & 49,8 & 61,4 \\
\hline $50-59$ & 182 & 53,3 & 41,7 & 52,2 \\
\hline $60 e+$ & 116 & 45,2 & 47,0 & 66,1 \\
\hline Escolaridade em anos & & $\mathrm{p}=0,002$ & $\mathrm{p}=0,007$ & $p=0,07$ \\
\hline Sem escolaridade & 46 & 32,6 & 32,6 & 56,5 \\
\hline $1-4$ & 465 & 55,5 & 52,4 & 60,7 \\
\hline $5-8$ & 503 & 60,8 & 54,9 & 67,7 \\
\hline+ de 8 & 91 & 52,2 & 42,2 & 58,9 \\
\hline Aplica agrotóxicos & & $\mathrm{p}<0,001^{*}$ & $\mathrm{p}<0,001^{\star}$ & $\mathrm{p}<0,001^{*}$ \\
\hline Não & 449 & 33,5 & 22,3 & 34,7 \\
\hline Até 2 dias/mês & 518 & 53,1 & 49,8 & 63,6 \\
\hline 3 e + dias/mês & 493 & 66,7 & 63,7 & 70,6 \\
\hline Prepara calda & & $\mathrm{p}<0,001^{*}$ & $\mathrm{p}<0,001^{\star}$ & $\mathrm{p}<0,001^{*}$ \\
\hline Não & 554 & 40,1 & 32,1 & 42,2 \\
\hline Até 2 dias/mês & 734 & 59,3 & 56,6 & 67,3 \\
\hline $3 e+$ dias/mês & 172 & 65,1 & 59,9 & 71,5 \\
\hline Onde trabalha com agrotóxico & & $p=0,06$ & $\mathrm{p}=0,03$ & $p=0,87$ \\
\hline Uma propriedade & 1.141 & 54,7 & 50,2 & 62,0 \\
\hline + de uma propriedade & 81 & 65,4 & 63,0 & 63,0 \\
\hline Orientação técnica & & $\mathrm{p}<0,001^{*}$ & $\mathrm{p}<0,001^{\star}$ & $\mathrm{p}<0,001^{*}$ \\
\hline Não & 297 & 32,0 & 26,3 & 46,8 \\
\hline Até 1 vez/ano & 205 & 58,5 & 56,6 & 66,8 \\
\hline+ de 1 vez/ano & 586 & 68,4 & 63,3 & 70,8 \\
\hline Total $^{\star \star}$ & 1.105 & 56,6 & 51,9 & 63,4 \\
\hline
\end{tabular}

* Tendência linear.

** Os totais das variáveis não incluem os valores ignorados.

maior risco, com uma taxa bruta de 6,1 episódios anuais para cada cem pessoas expostas. Os fatores ocupacionais que evidenciaram risco aumentado para intoxicações foram: aplicar agrotóxicos, re-entrar na cultura após aplicação, usar de equipamentos para trabalho com agrotóxicos mais que dez dias por mês e trabalhar com agrotóxicos em mais de uma propriedade (Tabela 4).

O uso de luvas mostrou associação com maior incidência de intoxicação. O uso de outros EPI, bem como, jornada de trabalho ou acesso a orientações técnicas não estiveram associadas a intoxicações (Tabela 4).

Observou-se um risco de intoxicações aumentado entre agricultores que trabalhavam em estabelecimentos onde era usada maior quantidade de fungicidas, sobretudo os tipos químicos ditiocarbamato e alaninato. Não foram evidenciadas diferenças em relação aos demais tipos químicos (Tabela 5).

\section{Discussão}


Intoxicações ao longo da vida conforme fatores sócio-demográficos, econômicos e produtivos.

Regressão de Poisson levando em consideração os anos de exposição aos agrotóxicos $(n=1.379)$.

Antônio Prado e Ipê, Rio Grande do Sul, Brasil, 1996.

\begin{tabular}{|c|c|c|c|c|}
\hline $\begin{array}{l}\text { Fatores demográficos } \\
\text { e econômicos }\end{array}$ & Taxa média $(\mathrm{dp})^{*}$ & $\begin{array}{c}\text { Prevalência } \\
\text { cumulativa }(\%)^{\star \star}\end{array}$ & $\begin{array}{c}\text { RR (IC95\%) } \\
\text { bruto }^{\star \star \star}\end{array}$ & RR ajustado ${ }^{\star \star \star \star}$ \\
\hline Total & $2,2(23,0)$ & 11,8 & & \\
\hline Sexo & & & $p=0,57$ & $p=0,42$ \\
\hline Masculino & $2,1(9,4)$ & 12,7 & 1 & 1 \\
\hline Feminino & $2,3(33,1)$ & 10,6 & $1,14(0,72-1,79)$ & $1,22(0,76-1,94)$ \\
\hline Idade (em anos) & & & $\mathrm{p}=0,10\left(0,07^{\star \star \star \star \star \star}\right)$ & $p=0,19\left(0,20^{\star \star \star \star \star \star}\right)$ \\
\hline $15-19$ & $1,9(7,3)$ & 6,2 & 1 & 1 \\
\hline $20-29$ & $5,0(55,1)$ & 8,6 & $0,65(0,23-1,80)$ & $0,78(0,27-2,22)$ \\
\hline $30-39$ & $1,7(7,5)$ & 20,4 & $0,66(0,30-1,48)$ & $0,68(0,28-1,64)$ \\
\hline $40-49$ & $1,2(4,9)$ & 24,7 & $0,41(0,20-0,85)$ & $0,45(0,21-0,95)$ \\
\hline $50-59$ & $2,5(10,8)$ & 24,1 & $0,58(0,27-1,23)$ & $0,68(0,31-1,51)$ \\
\hline $60 e+$ & $1,2(7,7)$ & 16,0 & $0,37(0,17-0,80)$ & $0,43(0,19-0,98)$ \\
\hline Escolaridade em anos & & & $p=0,21\left(0,07^{\star \star \star \star \star}\right)$ & $p=0,39\left(0,45^{\star \star \star \star \star}\right)$ \\
\hline Nenhuma & $0,2(0,8)$ & 5,2 & 1 & 1 \\
\hline $1-4$ & $1,6(7,9)$ & 14,6 & $2,71(0,91-8,11)$ & $2,48(0,82-7,51)$ \\
\hline $5-8$ & $3,0(33,8)$ & 10,7 & $3,30(1,08-10,11)$ & $2,56(0,85-7,71)$ \\
\hline $9 e+$ & $2,3(11,0)$ & 7,4 & $2,90(0,80-10,47)$ & $2,00(0,52-7,75)$ \\
\hline Renda bruta da produção & & & $p=0,10$ & $p=0,12\left(0,11^{\star \star \star \star \star \star}\right)$ \\
\hline Renda mais baixa & $3,9(43,3)$ & 9,8 & 1 & 1 \\
\hline Renda média baixa & $2,0(8,6)$ & 13,7 & $1,46(0,92-2,30)$ & $1,22(0,68-2,20)$ \\
\hline Renda média alta & $1,8(8,4)$ & 13,5 & $1,43(0,90-2,28)$ & $1,01(0,53-1,93)$ \\
\hline Renda mais alta & $0,9(4,5)$ & 9,8 & $1,00(0,60-1,66)$ & $0,60(0,31-1,14)$ \\
\hline Nível de mecanização & & & $p=0,28$ & $p=0,53\left(0,45^{\star \star \star \star \star}\right)$ \\
\hline Nenhum & $2,1(11,7)$ & 8,8 & 1 & 1 \\
\hline Pequeno & $3,9(45,5)$ & 11,4 & $1,66(0,82-3,34)$ & $1,33(0,56-3,17)$ \\
\hline Médio & $1,5(6,1)$ & 13,5 & $1,79(0,94-3,42)$ & $1,37(0,63-2,97)$ \\
\hline Grande & $1,4(8,7)$ & 9,4 & $1,26(0,60-2,64)$ & $0,92(0,34-2,50)$ \\
\hline Tem automóvel & & & $p=0,17$ & $p=0,09$ \\
\hline Não & $3,9(37,2)$ & 10,0 & 1 & 1 \\
\hline Sim & $1,6(6,9)$ & 12,1 & $1,37(0,87-2,16)$ & $1,47(0,94-2,28)$ \\
\hline
\end{tabular}

* Média de episódios ao longo da vida anos de exposição

x 100 - desvio padrão

** Prevalência Cumulativa (já teve algum episódio ao longo da vida).

*** Regressão de Poisson levando em consideração os anos de exposição.

$\star \star \star \star$ Variáveis ajustadas na regressão: idade, renda bruta da produção e ter automóvel.

$\star \star \star \star \star$ Valor de p para tendência linear.

O estudo foi metodologicamente criterioso, incluindo trabalho de campo com duração de cinco semanas, entrevistadores treinados, questionário pré-testado em estudo piloto, controle de qualidade e pequena proporção de perdas. A amostra foi ampla e representativa da agricultura familiar da região. Todos estes aspectos valorizam a qualidade das informações geradas e a dimensão dos resultados do estudo.

No entanto, o estudo pode ter sido afetado por causalidade reversa (por exemplo: EPI e intoxicações) viés de memória e de informação. Apesar do amplo número de entrevistados, a amostra pode ter sido insuficiente para revelar algumas associações com risco menos expressivo.

O critério para definir caso de intoxicação foi a informação referida pelo trabalhador. Este método já teve sua validade testada e reconhecida em vários estudos sobre agrotóxicos 13,14,15. Na região em estudo, a exposição era multiquí- 
Exposições ocupacionais e intoxicações por agrotóxicos $(n=1.379)$. Regressão de Poisson levando em consideração os anos de exposição aos agrotóxicos $(n=1.379)$. Antônio Prado e Ipê, Rio Grande do Sul, Brasil, 1996.

\begin{tabular}{|c|c|c|c|c|}
\hline Fatores ocupacionais & Taxa média $(\mathrm{dp})^{*}$ & $\begin{array}{c}\text { Prevalência } \\
\text { cumulativa }(\%)^{\star \star}\end{array}$ & $\operatorname{RR}(\mathrm{IC} 95 \%)^{\star \star \star}$ & RR ajustado ${ }^{\star \star \star \star}$ \\
\hline Total & $2,2(23,0)$ & 11,8 & & \\
\hline Aplica agrotóxicos & & $\mathrm{p}<0,001$ & $p=0,008$ & $\mathrm{p}=0,01\left(0,04^{\star \star \star \star \star}\right)$ \\
\hline Não aplica & $0,4(2,6)$ & 6,5 & 1 & 1 \\
\hline Até 3 dias/mês & $2,4(10,4)$ & 13,9 & $2,65(1,40-5,03)$ & $2,44(1,28-4,65)$ \\
\hline 3 dias e + & $3,7(37,8)$ & 14,4 & $2,49(1,34-4,65)$ & $2,44(1,30-2,56)$ \\
\hline Prepara a calda & & $\mathrm{p}=0,003$ & $p=0,17$ & $p=0,33\left(0,95^{\star \star \star \star \star}\right)$ \\
\hline Não prepara & $0,9(5,0)$ & 8,2 & 1 & 1 \\
\hline Até 3 dias/mês & $3,3(31,8)$ & 14,6 & $1,47(1,31-2,81)$ & $1,35(0,75-2,41)$ \\
\hline 3 dias e + & $1,6(8,7)$ & 11,3 & $0,92(0,84-2,55)$ & $0,93(0,44-1,95)$ \\
\hline Re-entrada após aplicação & & $p=0,004$ & $\mathrm{p}=0,02^{\star \star \star \star \star \star}$ & $\mathrm{p}=0,04\left(0,06^{\star \star \star \star \star}\right)$ \\
\hline Não faz isto & $0,9(4,3)$ & 9,1 & 1 & 1 \\
\hline Até 3 dias/mês & $5,6(48,3)$ & 15,6 & $1,89(1,12-3,17)$ & $1,87(1,09-3,21)$ \\
\hline 3 dias e + & $2,1(9,2)$ & 14,1 & $1,69(1,10-2,61)$ & $1,52(0,97-2,33)$ \\
\hline Onde lida com agrotóxicos & & $\mathrm{p}<0,001$ & $p=0,007$ & $\mathrm{p}=0,008$ \\
\hline Não lida/uma propriedade & $2,2(24,0)$ & 11,2 & 1 & 1 \\
\hline + de uma propriedade & $3,1(7,5)$ & 25,4 & $2,21(1,24-3,95)$ & $2,30(1,25-4,23)$ \\
\hline Equipamentos por agrotóxicos & & $\mathrm{p}<0,001$ & $p=0,01$ & $\mathrm{p}=0,01\left(0,11^{\star \star \star \star \star}\right)$ \\
\hline Não usa & $1,0(5,3)$ & 8,7 & 1 & 1 \\
\hline Usa até 10 dias/mês & $3,5(34,5)$ & 13,5 & $1,07(0,67-1,72)$ & $1,06(0,65-1,71)$ \\
\hline Usa + de 10 dias & $3,2(8,2)$ & 28,2 & $2,37(1,26-4,45)$ & $2,37(1,24-4,53)$ \\
\hline Jornada agrícola - safra & & $\mathrm{p}<0,001$ & $\mathrm{p}=0,24\left(0,14^{\star \star \star \star \star}\right)$ & $\mathrm{p}=0,31\left(0,19^{\star \star \star \star \star}\right)$ \\
\hline Até 8 horas/dia & $2,6(39,9)$ & 8,0 & 1 & 1 \\
\hline 9 a 12 horas/dia & $1,8(8,5)$ & 11,5 & $1,20(0,65-2,24)$ & $1,18(0,62-2,25)$ \\
\hline 13 horas/ dia ou + & $2,9(10,4)$ & 20,2 & $1,62(0,86-3,07)$ & $1,55(0,81-2,96)$ \\
\hline Usa luvas & & $p=0,05$ & $\mathrm{p}=0,001$ & $p=0,004$ \\
\hline Não & $1,1(4,3)$ & 10,8 & 1 & 1 \\
\hline Sim & $3,9(33,6)$ & 14,8 & $2,01(1,35-3,01)$ & $1,84(1,22-2,77)$ \\
\hline Usa máscaras & & $\mathrm{p}=0,001$ & $p=0,02$ & $p=0,07$ \\
\hline Não & $1,1(5,1)$ & 9,5 & 1 & 1 \\
\hline Sim & $4,1(34,8)$ & 16,3 & $1,72(1,09-2,71)$ & $1,58(0,96-2,61)$ \\
\hline Usa roupas de proteção & & $p=0,57$ & $p=0,92$ & $p=0,81$ \\
\hline Não & $1,6(6,7)$ & 12,3 & 1 & 1 \\
\hline Sim & $3,3(31,6)$ & 13,5 & $0,98(0,63-1,52)$ & $0,94(0,58-1,53)$ \\
\hline
\end{tabular}

* Média de episódios ao longo da vida anos de exposição

** Prevalência Cumulativa (já teve algum episódio ao longo da vida).

*** Regressão de Poisson levando em consideração os anos de exposição.

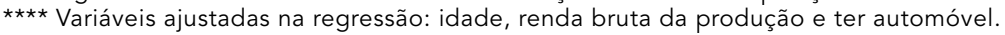

$\star \star \star \star *$ Valor de p para tendência linear. 
Tipos químicos usados no estabelecimento e intoxicações por agrotóxicos. Regressão de Poisson levando em consideração os anos de exposição aos agrotóxicos ( $n=1.379)$. Antônio Prado e Ipê, Rio Grande do Sul, Brasil, 1996.

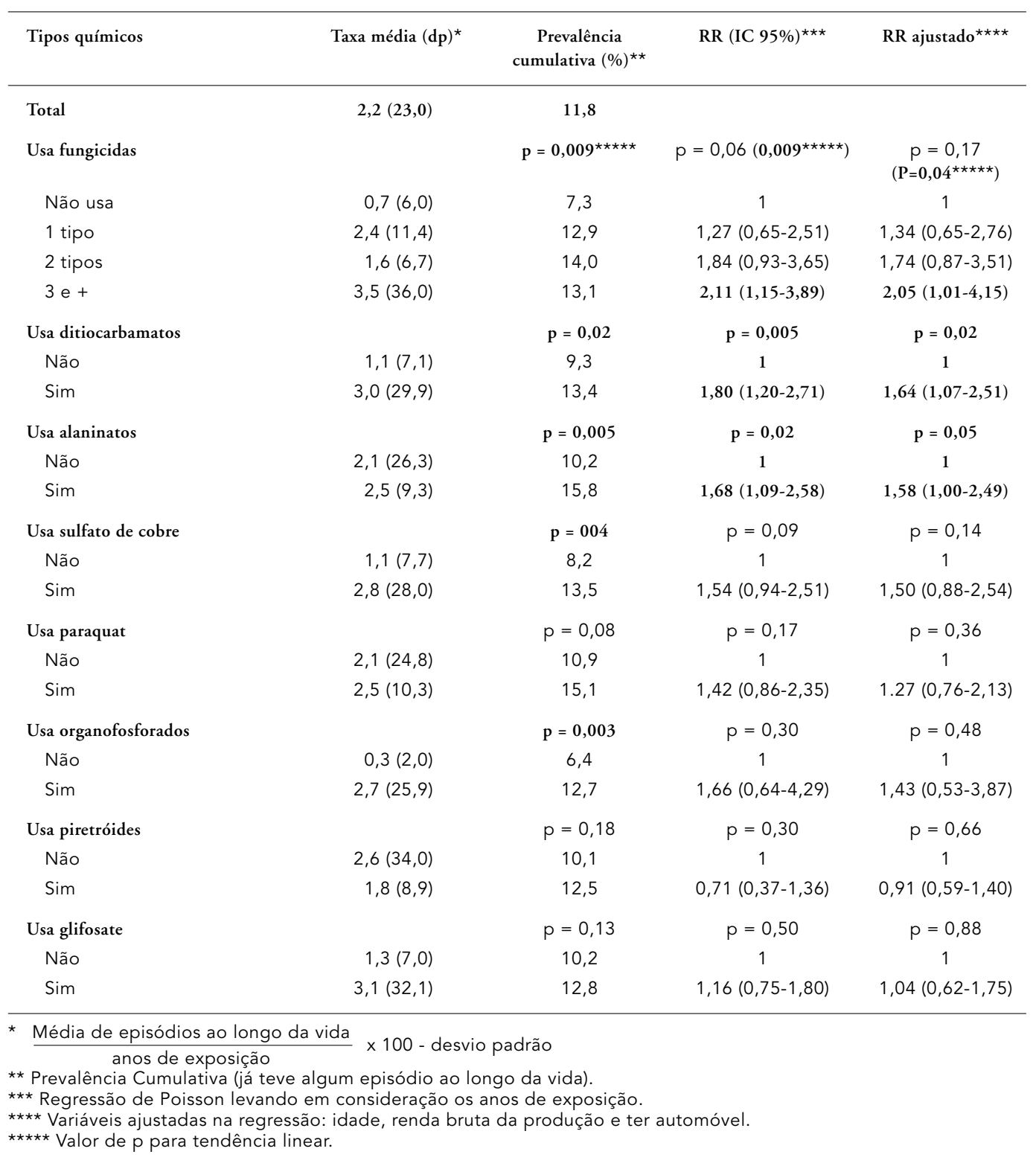

mica e a maioria dos produtos não dispunha de marcadores biológicos para utilização em área rural. Neste contexto, a informação referida permitiu uma boa aproximação da complexidade química do trabalho com agrotóxicos.

A freqüência de exposição foi bastante alta, em particular nas propriedades com uso intensivo de agrotóxicos, onde $86,0 \%$ dos trabalhadores costumavam ter exposição química. Este dado é próximo dos $83,0 \%$ encontrados na $\mathrm{Ni}$ carágua 2 , inferior aos $98,0 \%$ entre aplicadores licenciados de Minnesota 16 e superior aos 55,0\% encontrados entre agricultores asiáticos 17 .

Um pouco mais da metade dos trabalhadores relatou que costuma usar EPI, mas em relação à entrevista original, o controle de qualidade verificou uma superestimação do uso de EPI. Esta tendência na informação referida já foi detectada em outros estudos 18 e pode ter interferido na avaliação do efeito destas medidas de proteção. Em Iowa e Carolina do Norte, encontraram-se freqüências ainda menores de uso: respectivamente, 8,0 e 18,0\% usando máscaras para gases e 30,0 e $27,0 \%$ usando roupas 
impermeáveis e botas 19. Na pesquisa em quatro países da Ásia, foi encontrada grande variação nos dados sobre uso de máscaras $(9,0$ a $41,0 \%)$, luvas $(5,0$ a $95,0 \%)$ e de roupas específicas $(5,0$ a $67,0 \%) 17$.

Em Minas Gerais, os trabalhadores que não usavam medidas de proteção eram aqueles que estavam laboratorialmente mais intoxicados 5 . A associação, encontrada no presente estudo, entre uso de luvas e aumento na taxa de intoxicação, pode refletir causalidade reversa, ou se$\mathrm{ja}$, as pessoas passariam a se proteger mais após uma intoxicação. Além disto, o uso de EPI apresentou-se como uma espécie de marcador, sendo mais utilizado por pessoas com exposição química mais intensa.

A importância de proteger a pele é apontada em vários estudos que têm em vista a exposição dérmica como a principal via de absorção dos pesticidas 20,21,22. Nesta análise o uso de roupas de proteção não mostrou associação com as intoxicações, possivelmente devido ao viés de causalidade reversa ou a superestimação do uso de EPI. Além disto, existem autores que questionam a real eficácia destas roupas de proteção 17,23

No Sri Lanka, foi observado que, embora mais de $90,0 \%$ dos trabalhadores fossem conscientes sobre 9 entre 11 itens de proteção química, a grande maioria não usava EPI, em razão de desconforto e custos. Os autores destacaram o fato de o comportamento humano não ser determinado apenas pelo acesso a informações e apontaram outras estratégias, além de usar EPI, para reduzir os problemas com agrotóxicos como, por exemplo, o uso de métodos não-químicos para controle de pragas 3,18 .

$\mathrm{O}$ estudo conseguiu estimar a incidência anual de intoxicações ocupacionais por agrotóxicos durante 12 meses e ao longo da vida. Apesar de o delineamento ser transversal, os dados de exposição química e episódios de intoxicação foram colhidos de forma retrospectiva e analisados como uma coorte histórica. Assim, com base no número de episódios de intoxicações ao longo da vida e do número de anos de exposição por cem pessoas-ano, estimou-se a taxa de incidência 12. Esta taxa deve ser relativizada porque, por ter sido construída sobre um período muito extenso, pode estar afetada por viés de memória. Entretanto, a taxa anual estimada, de 2,2 episódios para cada cem trabalhadores expostos, mostrou-se consistente com os 2,0\% dos trabalhadores que relataram acidentes com agrotóxicos nos 12 meses anteriores à entrevista. A importância desta estimativa cresce ao se constatar o enorme sub-registro das intoxicações por pesticidas nas princi- pais fontes oficiais: CAT e SINITOX.

A taxa de incidência anual na Nicarágua, que construiu a estimativa de forma semelhante, foi 7,5 por cem pessoas-ano ${ }^{2}$. Na pesquisa feita em quatro países asiáticos, foram encontradas taxas anuais de 3,0 a 7,0\% de intoxicações 16 , sendo $7,0 \%$ no caso dos países com dados mais confiáveis 9 . Em outros estudos, as taxas anuais de intoxicações foram $7,5 \%$ no Sri Lanka 25, 9,0\% entre aplicadores de pesticidas da Indonésia 21 e $6,1 \%$ na Colômbia 24 . No presente estudo, como a taxa de incidência encontrada foi menor, este achado pode estar relacionado a menor toxicidade dos produtos predominantes na região e melhor nível sócio-econômico quando comparado a outros países em desenvolvimento.

A prevalência de $12,0 \%$ de intoxicações ao longo da vida é comparável às taxas encontradas na pesquisa em quatro países asiáticos, onde as intoxicações variaram de 12,0 a 19,0\% entre expostos 17. Selecionando a Malásia e o Sri Lanka, cujos dados eram de melhor qualidade, as prevalências foram 12,0 e 15,0\% 10. Na África do Sul, esta proporção foi de 9,0\% 25 e em outro estudo no Sri Lanka, variou de 7,0 a $22,0 \%$ conforme a região ${ }^{3}$.

As intoxicações foram associadas aos fungicidas (principalmente ditiocarbamato e alaninato) que eram também os produtos de uso mais freqüente na região. Boa parte das publicações aponta os inibidores de colinesterase (organo-fosforados-OF e carbamatos) como o grupo de produtos com maior risco de intoxicações agudas 8,9,26,27,28. O estudo envolvendo 35 mil aplicadores de pesticidas em Iowa e na Carolina do Norte encontrou predomínio de outros grupos químicos com destaque para glifosate, 2,4-D, triazinas e alachlor 19. Segundo a classificação toxicológica oficial (http://www4. anvisa.gov.br/agrosia/asp/default.asp, acessado em 20/Out/2003), estes fungicidas são considerados de menor toxidade aguda do que outros grupos químicos. No entanto, num contexto de alta exposição, o grupo dos fungicidas foi relacionado a um aumento na taxa de intoxicações agudas por agrotóxicos. Assim, destaca-se a necessidade dos serviços de saúde se estruturarem para a abordagem das intoxicações, dentro de um espectro químico mais amplo, que deve ser definido em consonância com os produtos predominantes em cada região.

As intoxicações ocorreram especialmente nos grupos que mais trabalhavam com pesticidas, sobretudo os grupos que aplicavam agrotóxicos mais de dez dias por mês e que trabalhavam com estes produtos em mais de uma propriedade. Este achado é consistente com outras 
pesquisas 2,21,23 e deve ser usado na definição dos alvos prioritários de ações preventivas.

Apesar do predomínio masculino em quase todas as formas de exposição, a participação feminina também mostrou-se expressiva, como mostra o fato de $51,0 \%$ delas atuarem como aplicadoras de agrotóxicos. No entanto, as mulheres usavam menos medidas de proteção química. Talvez por isso, não tenha sido encontrada diferença por sexo na incidência de intoxicações. Em outros estudos, o predomínio de exposição e intoxicações foi masculino 2,3,19 com exceção do estudo na Coréia, que apontou um aumento de 3,9 vezes no risco feminino de intoxicação 23

A escolaridade intermediária mostrou-se associada à maior exposição e uso de EPI. Escolaridade, acesso a orientações técnicas, bem como os demais indicadores econômicos e agrícolas não estiveram associados a intoxicações. A amostra pode ter sido insuficiente para captar riscos menos expressivos. Por outro lado, em virtude do modelo hegemônico de produção agrícola, o uso intensivo de agroquímicos ocorre justamente nos estabelecimentos mais produtivos e, portanto, com melhores indicadores econômicos. Desta forma, um possível efeito protetor oriundo das melhores condições sócio-econômicas (como cuidados de proteção) pode ter sido neutralizado pela exposição intensiva aos agrotóxicos.

Os empregados temporários eram contratados para suprir mão-de-obra, principalmente nas propriedades médias, que tinham alta

Resumo

O uso de agrotóxicos na agricultura brasileira é intenso e, apesar disso, são escassos os estudos de base populacional sobre as características da utilização ocupacional ou sobre as intoxicaçōes por agrotóxicos. Este estudo objetivou construir um perfil da exposição aos agrotóxicos e analisar a incidência de intoxicaçôes por estes produtos. Usando um delineamento transversal, foram avaliadas as características da propriedade e da exposição aos pesticidas. Entre 1.379 agricultores, a incidência anual de intoxicaçôes por agrotóxicos foi de 2,2 episódios por cem trabalhadores expostos, não sendo encontradas diferenças por sexo. Por meio de regressão de Poisson, evidenciou-se que entre as várias formas de exposição, aplicar agrotóxicos, re-entrar na cultura após aplicação e trabalhar com agrotóxicos em mais de uma propriedade se mostraram associadas a um aumento no risco de intoxicação. Os resultados deste estudo fornecem instrumentos para ações visando à redução das intoxicações ocupacionais por agrotóxicos.

Praguicidas; Exposiçāo a Pesticidas; Saúde Ocupacional; Incidência produtividade agrícola e uso intensivo de agrotóxicos. Este subgrupo relatou mais exposição química e uma freqüência de intoxicações maior que nos demais grupos, no entanto, o tamanho reduzido do grupo de empregados temporários $(\mathrm{n}=18)$ limita afirmações conclusivas sobre o tema.

Novos estudos mais aprofundados e com metodologias apropriadas para avaliar com maior precisão o quadro de intoxicações agudas por agrotóxicos, bem como estudos sobre morbidades crônicas relacionadas ao uso de pesticidas, colocam-se como prioridades para a área de pesquisa. Porém, algumas tendências na informação referida devem ser consideradas na compreensão dos resultados desta e de futuras pesquisas. No contexto da agricultura familiar, o agricultor tende a subestimar os dados sobre exposição ocupacional aos agrotóxicos e sobre intoxicações. Por outro lado, tende a superestimar as informações sobre o uso de medidas de proteção.

As entidades responsáveis pela proteção da saúde dos agricultores devem levar em conta os resultados deste estudo para o planejamento de suas atividades, visando à prevenção de novos casos de intoxicação por estes produtos. Também é importante apoiar a busca de novo modelo de produção agrícola, reduzindo a exposição química e melhorando a qualidade da vida durante o trabalho.

\section{Colaboradores}

N. M. X. Faria elaborou o projeto, coordenou a coleta de dados, realizou a análise de dados, revisão bibliográfica e redação do artigo. L. A. Facchini supervisionou a elaboração do projeto e apoiou a coleta de dados, supervisionou a análise de dados e revisou criticamente o rascunho do artigo e aprovou a versão final. A. G. Fassa supervisionou a elaboração do projeto e apoiou a coleta de dados, orientou o uso do programa Stata, supervisionou a análise de dados, e revisou criticamente o rascunho do artigo e aprovou a versão final. E. Tomasi supervisionou questões de informática e uso dos programas (Epi Info e SPSS) durante a estruturação do banco de dados e análise estatística, e também revisou criticamente o rascunho do artigo e aprovou a versão final. 
1. Dharmawardene LI. Pesticide poisoning among farmers in a health area in Sri Lanka. Ceylon Med J 1994; 39:101-3.

2. Keiffer M, McConnell R, Pacheco F, Daniel W, Rosenstock L. Estimating underreported pesticide poisoning in Nicaragua. Am J Ind Med 1996; 30:195-201.

3. van der Hoek W, Konradsen F, Athukorala K, Wanigadewa T. Pesticide poisoning: a major health problem in Sri Lanka. Soc Sci Med 1998; 46:495-504.

4. Faria NMX, Facchini LA, Fassa AG, Tomasi E. Processo de produção rural e saúde na Serra Gaúcha: um estudo descritivo. Cad Saúde Pública 2000; 16:115-28.

5. Soares W, Almeida RM, Moro S. Trabalho rural e fatores de risco associados ao regime de uso de agrotóxicos em Minas Gerais, Brasil. Cad Saúde Pública 2003; 19:1117-27.

6. Klein-Schwartz W, Smith GS. Agricultural and horticultural chemical poisonings: mortality and morbidity in the United States. Ann Emerg Med 1997; 29:232-8.

7. Litovitz TL, Klein-Schwartz W, Caravati EM, Youniss J, Crouch B, Lee S. Annual report of the American Association of Poison Control Centers Toxic Exposure Surveillance System. Am J Emerg Med 1999; 17:435-87.

8. Leveridge YR. Pesticide poisoning in Costa Rica during 1996. Vet Hum Toxicol 1998; 40:42-4.

9. London L, Ehrlich RI, Rafudien S, Krige F, Vurgarellis, P. Notification of poisoning in the Western Cape, 1987-1991. S Afr Med J 1994; 84:269-72.

10. Jeyaratnam J. Acute pesticide poisoning: a major global health problem. World Health Stat Q 1990; 43:139-44.

11. Faria NM, Facchini LA, Fassa AG, Tomasi E. Estudo transversal sobre saúde mental de agricultores da Serra Gaúcha (Brasil). Rev Saúde Pública 1999; 33:391-400.

12. Rothman KJ, Greenland S. Modern epidemioloy. Boston: Wilkins Publishers; 1998.

13. Murphy HH, Hoan NP, Matteson P, Abubakar AL. Farmers' self-surveillance of pesticide poisoning: a 12-month pilot in northern Vietnam. Int J Occup Environ Health 2002; 8:201-11.

14. Daniels JL, Olshan AF, Teschke K, Hertz-Picciotto I, Savitz DA, Blatt J. Comparison of assessment methods for pesticide exposure in a case-control interview study. Am J Epidemiol 2001; 153:1227-32.

15. Fritschi L, Siemiatycki J, Richardson L. Self-assessed versus expert-assessed occupational exposures. Am J Epidemiol 1996; 144:521-7.
16. Garry VF, Kelly JT, Sprafka JM, Edwards S, Griffith J. Survey of health and use characterization of pesticide appliers in Minnesota. Arch Environ Health 1994; 49:337-43.

17. Jeyaratnam J, Lun KC, Phoon WO. Survey of acute pesticide poisoning among agricultural workers in four Asian countries. Bull World Health Organ 1987; 65:521-7.

18. Sivayoganathan C, Gnanachandran S, Lewis J, Fernando M. Protective measure use and symptoms among agropesticide applicators in Sri Lanka. Soc Sci Med 1995; 40:431-6.

19. Alavanja MC, Sandler DP, McDonnell CJ, Lynch CF, Pennybacker M, Zahm SH, et al. Characteristics of pesticide use in a pesticide applicator cohort: the Agricultural Health Study. Environ Res 1999; 80 (2 Pt 1):172-9.

20. Levine RS, Doull J. Global estimates of acute pesticide morbidity and mortality. Rev Environ Contam Toxicol 1992; 129:29-50.

21. Kishi M, Hirschhorn N, Djajadisastra M, Satterlee LN, Strowman S, Dilts R. Relationship of pesticide spraying to signs and symptoms in Indonesian farmers. Scand J Work Environ Health 1995; 21: 124-33.

22. Machado Neto JG. Segurança no trabalho com agrotóxicos em cultura de eucalipto. São Paulo: Fundação de Estudos e Pesquisas em Agronomia, Medicina Veterinária e Zootecnia; 2001.

23. Shin DC, Kim HJ, Jung SH, Park CY, Lee SY, Kim CB. Pesticide poisoning and its related factors among Korean farmers. Med Lav 1998; 89 Suppl 2:S129-35.

24. Silva E, Morales L, Ortiz J. Evaluación epidemiológica de plaguicidas inhibidores de acetilcolinesterasa en Colombia, 1996-1997. Biomédica 2000; 20:200-9.

25. London L, Nell V, Thompson ML, Myers JF. Health status among farm workers in the Western Cape collateral evidence from a study of occupational hazards. S Afr Med J 1998; 88:1096-101.

26. Abdollahi M, Jalali N, Sabzevari O, Hoseini R, Ghnea T. A retrospective study of poisoning in Tehran. Clin Toxicol 1997; 35:387-93.

27. Xue DS-Z, Li Y, Jin ZG, Tao BG, Zhou QD. Trend of pesticide poisoning in recent decades in China. Med Lav 1998; 89:S99-104.

28. Litovitz TL, Klein-Schwartz W, Rodgers Jr. GC, Cobaugh DJ, Youniss J, Omslaer JC, et al. Annual report of the American Association of Poison Control Centers Toxic Exposure Surveillance System. Am J Emerg Med 2002; 20:391-452.

Recebido em 08/Dez/2003

Versão final reapresentada em 03/Mai/2004

Aprovado em 05/Mai/2004 